\title{
Factores de riesgo asociados con la prevalencia de hipertensión arterial en adultos de Bucaramanga, Colombia
}

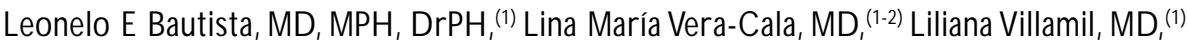 \\ Sandra M Silva, MD, (1) Iván M Peña, MD, (1) LauraV Luna, MD.(1)
}

\begin{abstract}
Bautista LE, Vera-Cala LM, Villamil L, Silva SM, Peña I, Luna LV.

Factores de riesgo asociados con la prevalencia de hipertensión arterial en adultos de Bucaramanga, Colombia. Salud Publica Mex 2002;44:399-405. El texto completo en inglés de este artículo está disponible en: http://www.insp.mx/salud/index.html
\end{abstract}

\section{Resumen}

Objetivo. Cuantificar la prevalencia de hipertensión arterial y su asociación con edad, sexo, estrato socioeconómico e índice de masa corporal. Material y métodos Encuesta transversal, de base poblacional, hecha en 1996, en una muestra aleatoria de 356 adultos $\geq 20$ años de edad, residentes en Bucaramanga, Colombia, en quienes se midieron peso, talla y presión arterial y se consideraron edad y sexo. Se usaron regresión lineal robusta y regresión binomial para estimar el efecto independiente de distintas variables so bre presión sistólica (PS), presión diastólica (PD) e hipertensión arterial (HTA). Resultados Las medias de PS y PD fueron significativamente mayores en hombres (122.5 y $88.1 \mathrm{mmH} \mathrm{g}$ ) que en mujeres (117.2 y $75.4 \mathrm{mmH}$ g). La PS ajustada aumento con la edad, mas en hombres que en mujeres, la PD no cambió significativamente con la edad. Por cada aumento de un $\mathrm{kg} / \mathrm{m}^{2}$ la PS y la PD aumentaron 0.76 y $0.69 \mathrm{mmH}$, respectivamente. Participantes de estrato socioeconómico bajo tuvieron una PS 6.8 y una PD $9.8 \mathrm{mmH}$ g mayor que los del nivel alto. La prevalencia ajustada de HTA (PHTA) aumentó con la edad siendo mayor en los $\geq 50$ años que en los de 20 a 30. El sexo no tuvo efecto sobre la PHTA $(p=0.795)$. Por cada aumento de un $\mathrm{kg} / \mathrm{m}^{2}$ la PHTA aumentó 3\% y las personas del estrato socioeconómico bajo tuvieron una PHTA 1.84 veces mayor que las del alto. Conclusiones La PHTA fue alta. Existe gran potencial para la prevención controlando el sobrepeso y la obesidad. Es necesario corroborar con nuevos estudios el aumento de la prevalencia en el estrato socioeconómico bajo e identificar sus causas. El texto completo en inglés de este artículo está disponible en: http://www.insp.mx/salud/index.html

Palabras clave: hipertensión; obesidad; índice de masa corporal; estrato socioeconómico; Colombia

\author{
Bautista LE, Vera-Cala LM,Villamil L, \\ Silva SM, Peña I, Luna LV. \\ Risk factors associated to the prevalence of hypertension \\ among adults in Bucamaranga, Colombia. \\ Salud Publica Mex 2002;44:399-405. \\ The English version of this paper \\ is available at: http://www.insp.mx/salud/index.html
}

\begin{abstract}
A bstract
Objective. To assess the prevalence of high blood pressure (PHBP) and its asso ciation with age, sex, socioeconomic status (SES), and body mass index $\left(\mathrm{BMI}=\mathrm{kg} / \mathrm{m}^{2}\right)$. Material and Methods. A cross-sectional survey was carried out in 1996, in a random sample of 356 adults aged 20 and older, residents of Bucaramanga, Colombia. W eight, height, and blood pressure readings were obtained, plus data on age and gender. Robust linear and log-binomial regression was used to estimate the independent effect of different risk factors on systolic (SBP) and diastolic (DBP) blood pressure and HBP. Results. Mean SBP and DBP were significantly higher in men (122.5 and $88.1 \mathrm{mmH}$ ) than in women (117.2 and $75.4 \mathrm{mmHg}$ ). The adjusted SBP increased with age, more in men than in women; DBP did not change with age. For each unit increase in BMI, SBP and DBP increased 0.76 and $0.69 \mathrm{mmH} \mathrm{g}$, respectively. SBP was 6.8 and D BP was $9.8 \mathrm{mmH}$ g higher in low-SES than in high-SES subjects. The adjusted PHBP increased with age and was higher in subjects $\geq 50$ years than in tho se 20 -30 years old. Sex had no significant effect on PHBP $(p=0.795)$. For each unit increase in BMI the PHBP increased $3 \%$, and low-SES subjects had a PHBP 1.84 times higher than high-SES subjects. Conclusions. A high PHBP was fo und.There is a great potential for prevention by weight control. Further studies are needed to confirm the increasing PHBP in low-SES subjects and to identify its causes. The English version of this paper is available at: http://www.insp.mx/salud/index.html
\end{abstract}

Key words: hyper tension; o besity; body mass index; socioeconomic status; Colombia

(1) Universidad Industrial de Santander, Bucaramanga, Colombia

(2) Programa de A poyo a Jóvenes Investigadores del Instituto Colombiano para el Desarrollo de la Ciencia y la Tecnología (Colciencias), Santa Fe de Bogotá, Colombia

Fecha de recibido: 12 de febrero de 2001 • Fecha de aceptado: 2 de abril de 2002

Solicitud de sobretiros: D ra. Lina María Vera Cala, Universidad Industrial de Santander, Facultad de Salud - Departamento de Salud Pública, Carrera 32, N 0. 29-31 Piso 3, Bucaramanga, Colombia. Correo electrónico: linamavc@ yahoo.com 
a hipertensión arterial (HTA) es uno de los principales factores de riesgo para el desarrollo de enfermedad cardiovascular. A pesar de que distintos factores se han asociado con la HTA, existe una gran heterogeneidad en la consistencia y magnitud de estas asociaciones de una población a otra.

Estudios transversales, ${ }^{1,2}$ y longitudinales, ${ }^{3,4}$ han mostrado relación positiva entre obesidad y presión arterial (PA). Sin embargo, algunos autores han sugerido que esta asociación no existe en mujeres de países no industrializados, $, 5,6$ ni en personas delgadas con bajo consumo de sal. ${ }^{78}$ En un estudio en siete países en desarrollo, ${ }^{1}$ en la población colombiana, a pesar de tener un índice de masa corporal (IMC) alto, no se encontró asociación entre éste y presión sistólica (PS).

En la mayoría de los estudios se ha identificado un aumento de la PA con la edad. Sin embargo, esta relación no se ha observado en poblaciones con bajos niveles de obesidad y estrés, baja ingesta de sal y fuerte actividad física ${ }^{9}$ y la magnitud del cambio de PA por año de edad parece depender del grado de modernización de la población. ${ }^{10,11}$ Esta relación tampoco fue observada en un estudio que incluía una muestra de trabajadores colombianos. ${ }^{1}$

Por otra parte, la prevalencia ajustada de hipertensión arterial (PHTA) en poblaciones occidentales es mayor en hombres que en mujeres de entre los 50 y 60 años de edad, pero esta relación se invierte en $\geq 50$ años; ${ }^{12,13}$ esto no se ha confirmado en poblaciones no occidentales, ${ }^{10,13,14}$ ni ha sido reportado en la población colombiana.

Por último, el riesgo de HTA y la PA parecen variar inversamente con indicadores socioeconómicos como la educación y el ingreso. ${ }^{15-17}$ No obstante, varios estudios muestran un riesgo de HTA similar, o aun mayor, en hombres de estrato socioeconómico (ESE) alto. ${ }^{14,18,19}$ Un informe en población colombiana mostró una relación inversa entre ESE y PS. ${ }^{1}$

Aunque existen reportes previos sobre la PHTA, ${ }^{1,20-22}$ nuestro conocimiento sobre los niveles de PA y el efecto de factores de riesgo asociados con la HTA en la población colombiana es limitado. En este estudio se investigó la distribución de la PA y la HTA y su relación con edad, sexo, obesidad y ESE en adultos residentes en Bucaramanga, Colombia. Esta información puede ser útil como base para planear estrategias preventivas y evaluar cambios futuros en los niveles de PA e HTA en esta población.

\section{Material y métodos}

Nuestros datos se recolectaron junto con los del Estudio Neuroepidemiológico Nacional de Colombia (ENN). ${ }^{23}$
En el ENN se seleccionaron al azar tres barrios de Bucaramanga, de estratos socioeconómicos bajo, medio y alto. Se actualizó el mapa cartográfico de cada barrio seleccionado y en cada uno se escogió al azar un número predeterminado de manzanas y viviendas. En cada vivienda se estudió a todos los residentes. La selección al azar se hizo por medio de números aleatorios generados por una computadora.

Todas las personas $\geq 20$ años que aceptaron participar $(n=356)$ fueron encuestadas y se les pesó, midió la talla y tomó la presión arterial. Se calculó el IMC y se clasificó a los participantes en peso normal (IMC $<25)$, con sobrepeso $(25 \leq \mathrm{IMC}<30)$ y obesos $(\mathrm{IMC} \geq 30)$ (24). La PA se midió siguiendo las normas de la Asociación Americana del Corazón. ${ }^{25}$ Dos observadores tomaron un total de tres medidas de la PA a cada participante, y el promedio de las dos últimas se usó en el análisis. Las personas con presión sistólica $\geq 140 \mathrm{mmHg}$ o diastólica $\geq 90 \mathrm{mmHg}$, y las que tomaban antihipertensivos fueron consideradas hipertensas. Cada persona fue clasificada dentro del ESE del barrio donde residía. Esta clasificación fue tomada del Departamento Nacional de Planeación ${ }^{26}$ y se basa en la disponibilidad de servicios y en la calidad de las viviendas en el barrio.

Debido a que en el $\mathrm{ENN}^{23}$ se sobremuestrearon las personas $\geq 50$ años, para obtener resultados aplicables a Bucaramanga, se hizo un ajuste directo de los promedios de acuerdo con la composición por edad y sexo de la población de la ciudad en $1993 .{ }^{27}$ El efecto independiente de distintos factores sobre la presión sistólica y diastólica, se exploró, en las personas que no estaban en tratamiento antihipertensivo, por medio de la regresión lineal múltiple robusta, con estimadores biponderados de Huber. ${ }^{28}$ Mientras que los efectos sobre la prevalencia ajustada de hipertensión arterial se estimaron usando la regresión binomial, que permite obtener prevalencias relativas (razones de prevalencia) de hipertensión ajustadas por otros factores de riesgo.$^{29}$ Los datos fueron analizados con el programa Stata. ${ }^{30}$

\section{Resultados}

Se estudiaron 356 personas con edad promedio de 48.5 años. El 39.9\% eran hombres. La media del IMC fue de $24.0 \mathrm{~kg} / \mathrm{m}^{2}$ (IC 95\%: 23.4 a 24.5), la prevalencia de sobrepeso fue de $25.5 \%$ (IC $95 \%$ : 20.6 a 30.4 ) y la de obesidad fue de $9.8 \%$ (IC 95\%: 5.8 a 13.8).

La PS aumentó con la edad, y tuvo una media de $122.5 \mathrm{mmHg}$ en hombres y de $117.2 \mathrm{mmHg}$ en mujeres (cuadro I). Sin embargo, en personas $<60$ años, la PS fue $8.6 \mathrm{mmHg}$ (IC 95\%: 4.1 a 13.0) mayor en hombres 
que en mujeres, mientras que en las $\geq 60$ años la PS fue similar en hombres y mujeres (diferencia $=-5.5 \mathrm{mmHg}$; IC 95\%: -15.0 a 4.0). Esta diferencia en el efecto de la edad sobre la PS en sujetos $<60$ y $\geq 60$ años fue estadísticamente significativa $(p<0.033)$.

Contrario a la presión sistólica, la relación entre presión diastólica y edad no fue monotónica. En ambos sexos, la PD aumentó con la edad hasta los 60 años, edad en la que empezó a disminuir (cuadro II). A pesar de que en general la media de PD en los hombres (81.1 $\mathrm{mmHg}$ ) fue mayor que en las mujeres (75.4 $\mathrm{mmHg}$ ), esta diferencia fue estadísticamente significativa (diferencia: $5.4 \mathrm{mmHg}$; IC 95\%: 2.3 a 8.4) en las personas $<60$ años, pero no en las $\geq 60$ años (diferencia: $1.1 \mathrm{mmHg}$; IC 95\%: -3.8 a 5.9). Esta interacción entre edad y sexo fue estadísticamente significativa $(p=0.007)$.
En el cuadro III se presentan los coeficientes de un modelo de regresión que resume los efectos del género, edad, IMC y ESE sobre la PS. El efecto de la edad sobre la PS dependió del sexo y viceversa. De acuerdo con el modelo, la media de la PS, ajustada por IMC y ESE, fue $8.2 \mathrm{mmHg}$ más alta en hombres $<60$ años (media ajustada=123.0 mmHg; IC 95\%: 121.8 a 124.2) que en mujeres $<60$ años (media ajustada $=114,7 \mathrm{mmHg}$; IC 95\%: 113.3 a 116.1). Sin embargo, en el grupo de $\geq 60$ años, la PS fue prácticamente igual en hombres (media $=130.4$; IC 95\%: 129.1 a 131.7) y mujeres (media= 130.3; IC 95\%: 128.7 a 131.9).

La PS también aumentó significativamente con el IMC. Por cada aumento de un $\mathrm{kg} / \mathrm{m}^{2}$ en el IMC la PS aumentó $0.76 \mathrm{mmHg}$ (cuadro III). Aunque el aumento no fue estadísticamente diferente en hombres y mujeres, modelos separados reflejaron un aumento de 1.01

Cuadro I

Promedios de presión arterial sistólica por grupos de edad y seXo. Bucaramanga, Colombia, 1996

\begin{tabular}{|c|c|c|c|c|c|c|}
\hline \multirow{2}{*}{$\begin{array}{l}\text { Edad } \\
\text { (años) }\end{array}$} & \multicolumn{2}{|c|}{ Hombres } & \multicolumn{2}{|c|}{ M ujeres } & \multicolumn{2}{|c|}{ Total } \\
\hline & Media & (IC 95\%) & Media & (IC 95\%) & Media & (IC 95\%) \\
\hline $20-29$ & 118.7 & $(115.4-122.0)$ & 107.6 & $(105.2-109.9)$ & 112.2 & $(109.9-114.5)$ \\
\hline $30-39$ & 118.7 & $(111.2-126.2)$ & 110.3 & $(106.4-114.2)$ & 113.1 & $(109.5-116.7)$ \\
\hline $40-49$ & 122.0 & $(112.6-131.4)$ & 118.3 & $(111.3-125.2)$ & 119.0 & $(113.3-124.7)$ \\
\hline $50-59$ & 130.3 & $(123.7-136.8)$ & 129.0 & $(122.4-135.5)$ & 129.5 & $(124.9-134.1)$ \\
\hline $60-69$ & 134.7 & $(127.0-142.3)$ & 137.7 & $(129.6-145.9)$ & 136.3 & $(130.8-141.8)$ \\
\hline $70+$ & 134.4 & $(125.5-143.4)$ & 149.2 & $(137.7-160.7)$ & 142.2 & $(134.7-149.6)$ \\
\hline Totala & 122.5 & $(118.5-126.4)$ & 117.2 & $(113.4-121.2)$ & 119.6 & $(117.7-121.6)$ \\
\hline
\end{tabular}

a:Ajustados por la distribución de edad y sexo de la población de Bucaramanga en 1993

Cuadro II

Promedios de presión arterial diastólica por grupos de edad y sexo. Bucaramanga, Colombia, 1996

\begin{tabular}{|c|c|c|c|c|c|c|}
\hline \multirow{2}{*}{$\begin{array}{l}\text { Edad } \\
\text { (años) }\end{array}$} & \multicolumn{2}{|c|}{ Hombres } & \multicolumn{2}{|c|}{ Mujeres } & \multicolumn{2}{|c|}{ Total } \\
\hline & Media & (IC 95\%) & Media & (IC 95\%) & M edia & (IC 95\%) \\
\hline $20-29$ & 76.3 & $(72.8-79.7)$ & 70.9 & $(67.9-73.9)$ & 73.1 & $(70.8-75.4)$ \\
\hline $30-39$ & 81.0 & $(75.1-86.9)$ & 74.7 & $(71.4-77.9)$ & 76.8 & $(73.8-79.7)$ \\
\hline $40-49$ & 90.7 & $(83.4-98.0)$ & 77.8 & $(73.2-82.3)$ & 80.3 & $(76.1-84.4$ \\
\hline $50-59$ & 83.2 & $(79.0-87.5)$ & 82.4 & $(78.8-86.0)$ & 82.8 & $(80.1-85.4)$ \\
\hline $60-69$ & 81.8 & $(76.8-86.8)$ & 81.7 & $(77.8-85.6)$ & 81.8 & $(78.7-84.8)$ \\
\hline $70+$ & 79.4 & $(75.0-83.8)$ & 77.9 & $(72.3-83.5)$ & 78.6 & $(75.2-82.1)$ \\
\hline Totala $^{a}$ & 81.1 & $(77.1-85.0)$ & 75.4 & $(71.5-79.3)$ & 78.0 & $(76.0-80.0)$ \\
\hline
\end{tabular}

a:Ajustados por la distribución de edad y sexo de la población de Bucaramanga en 1993 
$\mathrm{mmHg}$ (IC 95\%: 0.64 a 1.28 ) en los hombres y 0.58 $\mathrm{mmHg}$ (IC $95 \%$ : 0.06 a 1.09) en las mujeres. Por último, la PS aumentó $3.56 \mathrm{mmHg}$ en personas de ESE medio y $6.82 \mathrm{mmHg}$ en las de ESE bajo, en comparación con las de ESE alto.

Por otra parte, la PD estuvo asociada con sexo, IMC y ESE (cuadro IV). Una vez se ajustó por estas variables, la edad no tuvo un efecto significativo sobre la PD ( $p=0.126)$, ni hubo interacción entre edad y sexo $(p=0.109)$. Sin embargo, la PD promedio ajustada por edad, IMC y ESE en hombres (media $=80.2 \mathrm{mmHg}$; IC95\%: 79.4 a 81.0 ), fue $4.0 \mathrm{mmHg}$ (IC 95\%: 3.1 a 5.0) mayor que en mujeres (media $=76.2$; IC 95\%: 75.6 a 76.8).

Aunque no hubo diferencia significativa entre las personas de ESE medio y alto, las de ESE bajo tuvieron una PD $9.84 \mathrm{mmHg}$ mayor que las de ESE alto (cuadro IV). Por último, el aumento de la PD por $\mathrm{kg} / \mathrm{m}^{2}$ fue de $0.69 \mathrm{mmHg}$ y tendió a ser mayor en los hombres (media $=0.92$; IC $95 \%$ : 0.44 a 1.41) que en las mujeres (media=0.53; IC 95\%: 0.19 a 0.87).

La PHTA, ajustada por la distribución de edad y sexo en Bucaramanga en 1993, fue de $22.9 \%$ (IC 95\%: 18.6 a 27.3 ), $29.8 \%$ en hombres (IC 95\%: 21.7 a 37.8 ) y $17.2 \%$ en mujeres (IC 95\%: 12.8 a 21.6 ) y aumentó progresivamente con la edad, llegando a más de $40 \%$ en personas $\geq 60$ años. Sólo 30.6\% (IC 95\%: 22.0 a 40.2) de las personas con HTA estaban recibiendo tratamiento antihipertensivo.

Las estimaciones del efecto independiente de cada variable mostraron que la PHTA aumentó con la edad, llegando a ser casi siete veces mayor en personas $>70$ años que en las de 20 a 29 años (cuadro V).

\section{Cuadro III \\ Cambios ajustados en la presión arterial SISTÓLICA ASOCIADOS CON DISTINTAS CARACTERÍSTICAS de la persona. Bucaramanga, Colombia, 1996}

\begin{tabular}{lccc} 
Característica & $\begin{array}{c}\text { Cambio ajustado en la presión } \\
\text { arterial sistólica (mmHg) }\end{array}$ & $\begin{array}{c}\text { Intervalo de } \\
\text { confianza de 95\% }\end{array}$ & $\begin{array}{c}\text { Valor de } \\
P\end{array}$ \\
Constante & 74.26 & $63.59-84.94$ & 0.000 \\
\hline Edad & 0.51 & $0.39-0.64$ & 0.000 \\
\hline Hombre & 21.13 & $12.05-30.21$ & 0.000 \\
\hline Edad $\times$ Sexo* & -0.32 & $-0.50--0.14$ & 0.001 \\
\hline IMC $\left(\mathrm{kg} / \mathrm{m}^{2}\right)^{\ddagger}$ & 0.76 & $0.36-1.15$ & 0.000 \\
\hline ESE Alto & 1.00 & & \\
\hline ESE Medio & 3.56 & $-0.01-7.13$ & 0.051 \\
\hline ESE Bajo & 6.82 & $1.32-12.31$ & 0.015
\end{tabular}

* Interacción entre edad y sexo (femenino $=0$, masculino $=1$ )

* Indice de masa corporal

$\S$ Estrato socioeconómico, ESE alto es el grupo de referencia
Este aumento se manifestó principalmente a partir de los 40 años.

Por otra parte, cuando se tomaron en cuenta las demás variables, la PHTA no fue significativamente diferente en hombres y mujeres $(p=0.795)$. No obstante, la PHTA sistólica aislada (PS $\geq 160 \mathrm{mmHg}$ y $\mathrm{PD}<90$ ), ajustada por edad, IMC y ESE, fue casi tres veces ma-

\section{Cuadro IV}

Cambios ajustados en la presión arterial diastólica ASOCIADOS CON DISTINTAS CARACTERISTICAS

de la Persona. Bucaramanga, Colombia, 1996

\begin{tabular}{lccc} 
Característica & $\begin{array}{c}\text { Cambio ajustado en la presión } \\
\text { arterial diastólica }(\mathrm{mmHg})\end{array}$ & $\begin{array}{c}\text { Intervalo de } \\
\text { confianza de } 95 \%\end{array}$ & $\begin{array}{c}\text { Valor de } \\
p\end{array}$ \\
Constante & 54.64 & $48.69-62.59$ & 0.000 \\
\hline Edad & 0.05 & $-0.01-0.11$ & 0.126 \\
\hline Hombre & 3.60 & $1.26-5.93$ & 0.003 \\
\hline IMC $\left(\mathrm{kg} / \mathrm{m}^{2}\right)^{*}$ & 0.69 & $0.41-0.97$ & 0.000 \\
\hline ESE Alto & 1.00 & & \\
\hline ESE Medio & 1.24 & $-1.23-3.71$ & 0.986 \\
\hline ESE Bajo & 9.84 & $6.02-13.65$ & 0.000
\end{tabular}

* Indice de masa corporal

‡ Estrato socioeconómico, ESE alto es el grupo de referencia

\section{Cuadro V \\ Prevalencias relativas de hipertensión ajustadas PARA DISTINTAS CARACTERÍSTICAS DE LA PERSONA. Bucaramanga, Colombia, 1996}

$\begin{array}{cccc}\text { Característica } & \begin{array}{c}\text { Prevalencia relativa } \\ \text { de hipertensión }\end{array} & \begin{array}{c}\text { Intervalo de } \\ \text { confianza de } 95 \%\end{array} & \begin{array}{c}\text { Valor de } \\ \text { con }\end{array}\end{array}$

\begin{tabular}{cccc}
$\begin{array}{c}\text { Edad (años) } \\
20-29 *\end{array}$ & 1.00 & & \\
\hline $30-39$ & 1.56 & $0.56-4.37$ & 0.399 \\
\hline $40-49$ & 3.79 & $1.61-8.94$ & 0.002 \\
\hline $50-59$ & 5.08 & $2.28-11.31$ & 0.000 \\
\hline $60-69$ & 4.98 & $2.21-11.23$ & 0.000 \\
\hline $70-79$ & 6.88 & $3.11-15.19$ & 0.000
\end{tabular}

\begin{tabular}{llll} 
Hombres & 0.97 & $0.77-1.22$ & 0.795 \\
\hline IMC & 1.03 & $1.01-1.05$ & 0.000 \\
\hline Nivel Socioeconómico Alto & 1.00 & & \\
\hline Nivel Socioeconómico Medio & 1.15 & $0.86-1.53$ & 0.345 \\
\hline Nivel Socioeconómico Bajo & 1.84 & $1.45-2.35$ & 0.000 \\
& & & \\
a Grupo de comparación & & \\
₹ Indice de masa corporal $\left(\mathrm{Kg} / \mathrm{m}^{2}\right)$ & \\
Valor de p para ajuste del modelo basado en la prueba de Tsiatis: 0.954
\end{tabular}


yor en mujeres que en hombres ( $P R=2.72$; IC 95\%: 1.28 a $5.76 ; p=0.009$ ).

El aumento de la PHTA en personas de ESE bajo se mantuvo aún después de ajustar por sexo, edad e IMC (cuadro V). Aunque las personas de ESE medio tuvieron una prevalencia similar, las de ESE bajo tuvieron una prevalencia $84 \%$ mayor que las de ESE alto $(p<0.001)$.

Por cada aumento de un $\mathrm{kg} / \mathrm{m}^{2}$ en el IMC, la PHTA aumentó 3\% (IC 95\%: 1\% a 5\%) (cuadro V). Sin embargo, los efectos del peso y la talla como variables aisladas fueron de sentido contrario. Mientras que por cada aumento de cinco $\mathrm{kg}$ de peso la PHTA aumentó 1.06 veces (IC 95\%: 1.02 a 1.10), por cada aumento de $10 \mathrm{~cm}$ de estatura se redujo en un factor de 0.83 veces (IC 95\%: 0.70 a 0.98$)$. Además, las personas con sobrepeso no tuvieron una prevalencia significativamente mayor de HTA (prevalencia relativa, $P R=1.27$, IC $95 \%$ : 0.90 a 1.78 ), mientras que las personas obesas tuvieron una prevalencia más de dos veces mayor que las personas normales ( $P R=2.17$ IC 95\%: 1.66 a 2.85). Teniendo en cuenta que $25.5 \%$ de la población tenía sobrepeso y $9.8 \%$ tenía obesidad, $16.7 \%$ de los casos de HTA podrían atribuirse a estas características.

\section{Discusión}

Nuestros resultados reflejan una alta PHTA en Bucaramanga, particularmente en personas $\geq 60$ años. Las prevalencias observadas fueron similares a las de una encuesta en más de 14000 voluntarios $\geq 20$ años de Santander: $21.4 \%$ en la población general, $25.0 \%$ en hombres y $18.4 \%$ en mujeres. Sin embargo, estudios en otros subgrupos de la población colombiana han reflejado prevalencias que varían de $12.0 \%{ }^{21}$ a $33.9 \%{ }^{22}$

Una vez se ajustó por edad, IMC y ESE, la PHTA fue similar en hombres y mujeres. Sin embargo, la HTA sistólica aislada fue tres veces más frecuente en mujeres que en hombres, lo cual es consistente con lo hallado en otras poblaciones occidentales. ${ }^{31,32}$

Como ocurre en otras poblaciones, ${ }^{33}$ las presiones sistólica y diastólica fueron mayores en hombres que en mujeres $<60$ años y similares después de esta edad. La caída en el aumento de la PD con la edad después de los 60 años, con aumento progresivo de la PS, también ha sido observado en otras poblaciones ${ }^{34} \mathrm{y}$ es probable que no se deba a un sesgo de sobrevivencia ni a un agotamiento de la HTA diastólica, sino más bien a un aumento del endurecimiento de las arterias de gran tamaño.

En el estudio INTERSALT, ${ }^{20}$ llevado a cabo hace más de una década, la PS promedio de la población colombiana fue muy similar, pero la PD promedio fue significativamente menor que la estimada en nuestro estudio (119.1 vs $119.6 \mathrm{mmHg}$ para PS y $66.6 \mathrm{mmHg}$ vs $78.0 \mathrm{mmHg}$ para $\mathrm{PD}$, respectivamente). Este aumento de la PD podría deberse al envejecimiento de la población. Sin embargo, el hecho de que la PS promedio no haya cambiado y que la PD disminuye después de los 50 a 60 años de edad, sugiere que es posible que el aumento se deba a otras causas. La elevación de la PD promedio en la población explicaría el aumento en la PHTA: de $17.1 \%$ en INTERSALT ${ }^{20}$ a $22,9 \%$ en nuestro estudio.

Diversos estudios han mostrado que el peso corporal está positivamente asociado con la PA (2.70.330.34). En nuestro estudio, los cambios en PS y PD por cada aumento de $10 \mathrm{~kg}$ de peso (3.42 y 2.10 $\mathrm{mmHg}$ por $\mathrm{kg} / \mathrm{m}^{2}$, respectivamente) fueron similares a los notificados por Dyer ${ }^{2}$ en INTERSALT: $3.0 \mathrm{mmHg}$ en PS y entre 2.0 y $2.7 \mathrm{mmHg}$ en $\mathrm{PD}$, pero menores que los observados por $\mathrm{He}^{35}$ en un estudio en personas delgadas de una zona rural de China: entre 3.5 y $5.8 \mathrm{mmHg}$ para la PS y 2.4 y 5.2 para PD. ${ }^{35}$ Estas diferencias sugieren que el aumento de la PA asociado con la obesidad es mayor en individuos delgados que en obesos.

Por cada aumento de un $\mathrm{kg} / \mathrm{m}^{2}$ en el IMC hubo un aumento de $0.76 \mathrm{mmHg}$ en la PS y de $0.69 \mathrm{mmHg}$ en la PD. Cambios similares fueron observados en INTERSALT: ${ }^{2}$ entre 0.70 y 0.94 en la PS y entre 0.46 y 0.76 en la PD. En general, nuestros datos reflejaron un mayor efecto del IMC sobre la presión sistólica que sobre la presión distólica, y en los hombres que en las mujeres, tal y como se ha observado en otros estudios. $2,35,36$

Aunque el mecanismo de la asociación entre IMC y PA no se ha establecido, se sabe que la obesidad aumenta tanto la resistencia a la insulina como la hiperinsulinemia, cambios que pueden llevar a un incremento de la termogénesis, mediado simpáticamente para restablecer el balance energético. ${ }^{37}$ Este aumento de la actividad simpática estimula al corazón, los vasos sanguíneos y el riñón, contribuyendo a la HTA. ${ }^{37}$

Un estado de inflamación sistémica crónica ligera podría también explicar el mayor riesgo de hipertensión observado en personas obesas. La obesidad se asocia con un estado crónico de inflamación sistémica moderada que se expresa en niveles elevados de proteina C-reactiva, interleuquina-6 y TNF-a. ${ }^{38,39}$ Este estado inflamatorio se asocia con una disminución de la vasodilatación arterial dependiente del endotelio, ${ }^{40,41}$ una condición que a su vez está asociada con la presencia de hipertensión arterial. ${ }^{42,43}$ Finalmente, estudios recientes sugieren que un estado de inflamación crónica moderada puede ser un factor de riesgo para desarrollar HTA..$^{44,45}$ 
El hallazgo de una mayor prevalencia de HTA en personas de ESE bajo no ha sido reportado en la población colombiana, aunque sí en otras occidentales. ${ }^{15-17}$ Este resultado es más llamativo si se toma en cuenta que es probable que la asociación ESE-HTA esté disminuida por error de clasificación no diferencial en la medición del ESE. Esta asociación podría estar mediada por la educación, factores ambientales y dietéti$\cos$, y existe evidencia de que no es el resultado de una mayor susceptibilidad a factores de riesgo tradicionales en personas de ESE bajo. ${ }^{46}$ Esto sugiere que existen factores de riesgo para HTA aún no identificados cuya prevalencia es mayor en las personas de ESE bajo que en las de alto.

A pesar de que nuestro estudio se basa en una muestra aleatoria de la población y de que se tomó información de buena calidad, algunos factores de riesgo para HTA, como el consumo de alcohol y la ingesta de sal, no pudieron ser medidos debido a la falta de instrumentos apropiados para hacer tales mediciones en esta población.

En síntesis, el patrón de asociación de la hipertensión arterial con edad, sexo, estrato socioeconómico e índice de masa corporal fue similar al observado en estudios en países desarrollados. Nuestros resultados sugieren un gran potencial para la reducción de la prevalencia de hipertensión arterial y sus efectos sobre la morbimortalidad por enfermedades cardiovasculares, por medio de la prevención y control del sobrepeso. Además, es necesario conducir nuevos estudios con el fin de corroborar el aumento de la prevalencia de hipertensión arterial en personas de estrato socioeconómico bajo e identificar factores asociados con dicho aumento.

\section{Referencias}

1. IN CLEN Multicentre Collaborative Group. Risk factors for cardiovascular disease in the developing world.A multicentre collaborative study in the International Clinical Epidemiology N etwork. J Clin Epidemiol 1992;45(8):841-847.

2. Dyer AR, Elliot P, Shipley M for the IN TERSALT Cooperative Research $G$ roup. Body mass index versus high and weight in relation to blood pressure: Findings for the 10079 persons of the IN TERSA LT Study.Am J Epidemiol 1990;131(4):589-596.

3. Srinivasan SR, Bao W, W attigney W A, Berenson GS. Adolescent overweight is associated with adult overweight and related multiple cardiovascular risk factors: The Bogalusa Heart Study. Metabolism 1996 Feb;45(2):235-240.

4. N eser W B, Thomas J, Semenya K, Thomas DJ, Gillum RF. 0 besity and hypertension in a longitudinal study of black physicians:The Meharry cohort study. J Chronic Dis 1986;39(2):105-113.
5. Walker AR, Walker BF, Manetsi B, Tsotesi N G, W alker AJ. 0 besity in black women in Soweto, South Africa: Minimal effects on hypertension, hyperlipidaemia and hyperglycaemia.J R Soc Health 1990;110(3):101-103. 6. W alker ARP, W alker BF, Manetsi B, Molefe 0 , W alker AJ, Vorster $\mathrm{HH}$. $O$ besity in indigent elderly rural African women: Effects on hypertension, hyperlipidaemia and hyperglycaemia. Int JVitam N utr Res 1990;61(3): 244-250.

7. Dyer AR, Elliot P.The IN TERSALT Study: Relation of body mass index to blood pressure. IN TERSALT Cooperative Research G roup.J Hum Hypertens 1989;3(5):299-308.

8. Poulter N, Khaw KT, Hopwood BE, Mugambi M, Peart W S, Rose G et al. Blood pressure and its correlates in an African tribe in urban and rural environments. J Epidemiol Community Health 1984;38(3):181-186.

9. Shaper AG. Communities without hypertension. En: Shaper AG, Hutt MSR, Feifar Z, ed. Cardiovascular disease in the tropics. London: British Medical Association, 1974:77-83.

10. He J, Klag MJ,W helton PK, C hen J-Y, Mo J-P, Q uian MC et al. Migration, blood pressure pattern, and hypertension:The Yi Migrant Study.Am J Epidemiol 1991;134(10):1085-1101

11. Cooper R, Rotimi C ,Ataman S, McG ee D, 0 sotimehin B, Kadiri S et al. The prevalence of hypertension in seven populations of W est African origin. Am J Public Health 1997;87(2):160-168.

12. D rizd T, D annenbergAL, Engel A . Blood pressure levels in persons 1874 years of age in 1976-80, and trends in blood pressure from 1960 to 1980 in the United States.Vital Health Stat 1986;11(234):1-68.

13. Dustan HP. Gender differences in hypertension. J Hum Hypertens 1996;10(6):337-340.

14. Singh RB, Beegom R, Ghosh S, Niaz MA, Rastogi V, Rastogi SS et al. Epidemiological study of hypertension and its determinants in an urban population of N orth India.J Hum Hypertens 1997;11(10):679-685.

15. Hypertension Detection and Follow-up Program Cooperative Group. Race, education and prevalence of hypertension. Am J Epidemiol 1977;106(5):351-361.

16. Stamler R, Shipley M, Elliot P, Dyer A, Sans S, Stamler J. Higher blood pressure in adults with less education: Some explanations from IN TERSALT. Hypertension 1992;19(3):237-241.

17. Hazuda HP. Hypertension in the San Antonio Heart Study and the Mexico City Diabetes Study: Sociocultural correlates. Public Health Rep 1996;111(Suppl 2):18-21.

18. McKetney EC, Ragland DR. Henryism J. Education, and blood pressure in young adults. The CARD IA study. C oronary Artery Risk D evelopment in Young A dults Study. Am J Epidemiol 1996;143(8):787-791.

19. Siziya S, Marufu T, Matchaba-H ove RB. Relationship of causal blood pressure to smoking, education and occupation in a high density town near Harare, Zimbabwe. Cent Afr J Med 1995;41(7):219-224.

20. IN TERSALT Cooperative Research G roup.IN TERSALT:An international study of electrolyte excretion and blood pressure: Results for 24 hour urinary sodium and potassium excretion. BMJ 1988;297(6644):319-328.

21. N ewman A, Herrera JA. Prevalencia de hipertensión arterial y el estrés psicosocial en una comunidad de Cali. Colombia Med 1993;24(2):4446.

22. $\mathrm{N}$ aranjo P. Hipertensión y prevalencia de algunos factores de riesgo en área rural de San Pedro,Antioquia 1994. Boletín Epidemiológico de Antioquia 1995;XX:42-45.

23. Pradilla G, Rosselli D, Bautista L, Morillo L, U ribe C , TakeuchiY et al. Estudio neuroepidemiológico nacional. Bucaramanga, Colombia: Ministerio de Salud - Universidad Industrial de Santander, 1995.

24. Seidell JC, Flegal KM.Assessing obesity: C lassification and epidemiology. Br Med Bull 1997;53(2):238-252.

25. Perloff D, G rim C, Flack J, Frohlich ED, Hill M, McD onald M et al. Human blood pressure determination by sphygmomanometry. Circulation 1993;88(5 Pt 1):2460-2470. 
26. Departamento $\mathrm{N}$ acional de Planeación. Estratificación socioeconómica. Manual general. Cabeceras municipales Tipo 3 y localidades 0 centros poblados hasta con tres mil habitantes. Santafé de Bogotá: D N P 1994.

27. Gao S, Hui SL, Hall KS, Hendrie HC. Estimating prevalence from twophase surveys with non'response at the second phase. Stat Med 2000;19:2101-2114.

28.W eisberg S.Applied linear regression. 2nd ed. N ueva York: John W iley \& Sons, 1985:252-253.

29.W acholder S. Binomial regression in GLIM: Estimating risk ratios and risk differences. Am J Epidemiol 1986;121(1):174-184.

30. StataC orp. Stata Statistical Software: Release 6.0. College Station,TX: Stat Corporation, 1999.

31.W ilking SV, Belanger A, Kannel W B, D 'A gostino RB, Steel K. D eterminants of isolated systolic hypertension. JA MA 1988;260(23):3451-3455.

32. van den Ban GC, Kampman E, Schouten EG, Kok FJ, van der Heide RM, van der Heide-Wessel $C$ et al. Isolated systolic hypertension in Dutch middle age and all-cause mortality:A 25-year prospective study. Int J Epidemiol 1989;18(1):95-99.

33. Franklin SS, Gustin W 4th, W ong N D, Larson MG, W eber MA, Kannel W B et al. Hemodynamic patterns of age-related changes in blood pressure.The Framingham Heart Study. Circulation 1997:96(1):308-315.

34. Stamler R, Stamler J, Riedlinger W F,Algera G, Roberts RH.W eight and blood pressure. Findings in hypertension screening of 1 million Americans. JAMA 1978;240(15):1607-1610.

35. He J, Klag MJ,W helton PK, C hen JY, Q ian MC, He G Q . Body mass and blood pressure in a lean population in Southwestern C hina.Am J Epidemiol 1994;139(4):380-389.

36. Kaufman J, A suzu M, Mufunda J, Forrester T, W ilks R, Luke A et al. Relationship between blood pressure and body mass index in lean populations. Hypertension 1997;30(6):1511-1516.
37. Raven GM, Chen YD. Insulin resistance, its consequences, and coronary heart disease. Must we choose one culprit? Circulation 1996;93(10): 1780-1783.

38.Visser M, Bouter LM, MCQ uillan GM,W ener MH, HarrisTB. Low-grade systemic inflammation in overweight children. Pediatrics 2001; 107: E13. 39.Yudkin JS, Stehouwer CD, Emeis JJ, C oppack SW. C-reactive protein in healthy subjects:Associations with obesity, insulin resistance, and endothelial dysfunction: A potential role for cytokines originating from adipose tissue? Arterioscler Thromb Vasc Biol 1999;19:972-978.

40. Sinisalo J, Paronen J, Mattila KJ, Syrjala M, A lfthan G, Palosuo T et al. Relation of inflammation to vascular function in patients with coronary heart disease.Atherosclerosis 2000;149:403-411.

41. Hingorani AD, C ross J, Kharbanda RK, Mullen MJ, Bhagat K, Taylor M et al. Acute systemic inflammation impairs endothelium-dependent dilatation in humans. Circulation 2000;102:994-999.

42. Panza JA, Q uyyumi AA, Brush JE Jr, Epstein SE.A bnormal endotheliumdependent vascular relaxation in patients with essential hypertension. $\mathrm{N}$ Engl J Med 1990;323:22-27.

43. Liyama K, N agano M, Yo Y, N agano N , Kamide K, Higaki J et al. Impaired endothelial function with essential hypertension assessed by ultrasonography. Am Heart J 1996;132:779-782.

44. Bautista LE, López-Jaramillo P,Vera LM, C asas JP, 0 tero AP, Guaracao Al. Is $C$-reactive protein an independent risk factor for essential hypertension? J Hypertens 2001;19:857-861.

45. Chae CU, Lee RT, Rifai N , Ridker PM. Blood pressure and inflammation in apparently healthy men. Hypertension 2001 sep;38(3):399-403.

46. N ilsson P, 0 stergren PO, Lindholm L, Schersten B. Can social class differentials in hypertension be explained by the general susceptibility hypothesis? Soc Sci Med 1994;38:1235-1242. 